\title{
Fluorescence signalling of the transition metal ions: Design strategy based on the choice of the fluorophore component
}

\author{
N B SANKARAN, S BANTHIA and A SAMANTA* \\ School of Chemistry, University of Hyderabad, Hyderabad 500 046, India \\ e-mail: assc@uohyd.ernet.in
}

\begin{abstract}
Transition metal ions are notorious for their fluorescence quenching abilities. In this paper, we discuss the design strategies for the development of efficient off-on fluorescence signalling systems for the transition metal ions. It is shown that even simple fluorophore-spacer-receptor systems can display excellent off-on fluorescence signalling towards the quenching metal ions when the fluorophore component is chosen judiciously.
\end{abstract}

Keywords. Fluorosensor; transition metal ions; fluorescence enhancement.

\section{Introduction}

Because of possible application as molecular switches or devices in information processing and computation, there is a great deal of current interest in molecular systems capable of performing light-induced logic operations. ${ }^{1-3}$ A variety of molecular photonic devices that have been developed in recent years are essentially assemblies of suitably organized molecular components capable of performing some logic function characteristic of the assembly. ${ }^{4-17}$ Of particular interest are the fluorosensors, molecular devices powered by light and the response of these systems is based on fluorescence. Fluorosensors are generally multi-component systems comprising a signalling moiety (fluorophore) and a guest-binding site (commonly referred to as receptor); the two are often separated by a spacer group. ${ }^{4}$ The components are chosen such that the communication between the receptor and fluorophore results in 'switching off' of the fluorescence of the system in the absence of a guest. However, in presence of a guest, the communication between the receptor and fluorophore gets turned-off leading to the recovery of the fluorescence of the system. Thus, the presence of a guest is indicated by switching on of the fluorescence or fluorescence enhancement (FE). Photoinduced intramolecular electron transfer (PIET) is the most commonly exploited mechanism of communication between the terminal moieties of a fluorophore-spacer-receptor system. ${ }^{4}$ The design principle of the off-on fluorescence signalling of a guest is schematically illustrated in scheme 1.

The fluorosensors for the metal ions based on PIET-mechanism commonly employ an amine as the receptor. While a variety of metal ions can be sensed by fluorophorespacer-receptor systems employing simple amines as receptors, ${ }^{10}$ it is reported, however, that with such systems no significant FE is possible in the presence of the transition metal ions ${ }^{4}$ because of their quenching nature. ${ }^{18,19}$ One needs to specially design a

*For correspondence 
Noguest:

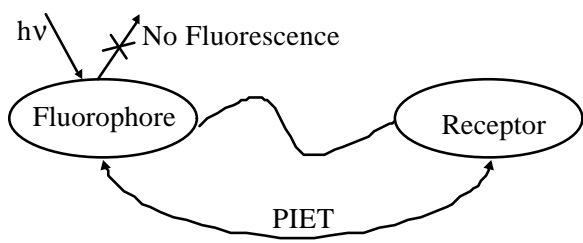

Presence of a guest:

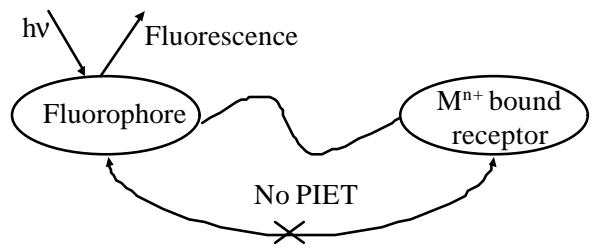

Scheme 1.

receptor for these quenching metal ions such that the metal ions in the bound condition are not accessible to the fluorophore for quenching. One such success has recently been reported by Ghosh et al ${ }^{14}$ where a cryptand receptor has been employed to trap the metal ions in the cavity so as to suppress the quenching influence of the metal ions. Based on this receptor design approach, Ghosh et al have developed a fluorophore-spacer-receptor system that shows fluorescence enhancement even in the presence of the quenching metal ions. The ability of this system to function as an off-on fluorosensor for the transition metal ions is attributable to the special topology of the receptor. The receptor, a cryptand moiety in this case, provides a cavity wherein the quenching metal ions are trapped and thus become unavailable for fluorescence quenching. In other words, in this approach, the metal ion-receptor interaction has been increased to reduce indirectly the undesired communication between the metal ion and fluorophore that leads to fluorescence quenching.

While the above approach is indeed an elegant one, it requires considerable synthetic skill for the development of fluorosensors of this kind. Further, the systems so developed are fairly complex. Since the quenching interaction between the fluorophore and the metal ions is known to be predominantly redox in nature, ${ }^{18,19}$ we thought that it might be possible to reduce this interaction simply by making the fluorophore electronically deficient by suitable substitution or simple structural modifications. It should be noted that such modification not only minimizes the undesired fluorophore-metal ion quenching interaction in the metal-bound state, but also enhances the desired PIET communication between the fluorophore and the receptor in the unbound state; both favouring an increase in the efficiency of the sensor system. In this paper, we present fluorescence signalling behaviour of some of the systems developed ${ }^{15-17}$ following the above design logic. The results suggest that it is indeed possible for even simple fluorophore-spacer-receptor systems to exhibit exceptionally high fluorescence enhancement in the presence of quenching metal ions. 


\section{Materials and methods}

All the systems reported in this manuscript have been synthesised and fully characterised in our laboratory following procedures described elsewhere. ${ }^{15-17}$ Hydrated perchlorate salts of the transition metal ions have been used for studying the influence of the metal ions. The fluorescence measurements were carried out by using $\sim 10^{-5} \mathrm{M}$ solutions of the compounds. The effect of the metal ions on the fluorescence intensity was examined by adding a few microlitres of a stock solution of the metal ions to a known volume (usually $2 \mathrm{ml}$ ) of the solution of the probe molecule. The addition was limited to $100 \mu \mathrm{l}$ such that the volume change was not significant. The concentration of the metal salts was varied between $5 \times 10^{-6}-5 \times 10^{-3} \mathrm{M}$. The solvents used in the measurements, tetrahydrofuran and acetonitrile, were rigorously purified following standard procedures.

The absorption and fluorescence spectra of the systems were recorded on Shimadzu spectrophotometer (UV-3101PC) and Spex spectrofluorimeter (Fluoromax-3) respectively. All fluorescence spectra were corrected for the instrumental response. Timeresolved fluorescence behaviour of the systems was studied on a single photon counting spectrofluorimeter (IBH, Model 5000). The instrument was operated with a thyratrongated flash lamp filled with hydrogen at a pressure of $0.5 \mathrm{~atm}$ and the pulse width of the lamp under the operating condition was $\sim 1.2 \mathrm{~ns}$. The lifetimes were estimated from the measured fluorescence decay curves and the lamp profile using a nonlinear least-squares iterative fitting procedure. The cyclic voltammetric measurements were carried out with Cypress Model CS-1090/CS-1087 computer controlled electroanalytical system. Ag/ $\mathrm{AgCl}$ was used as the reference electrode, a glassy carbon as the working electrode and a $\mathrm{Pt}$ wire as the auxiliary electrode. The redox potentials were measured in $\mathrm{N}_{2}$ bubbled acetonitrile using $0 \cdot 1 \mathrm{M}$ TBAP as the supporting electrolyte. The scanning speed was maintained at $100 \mathrm{mV} / \mathrm{s}$.

\section{Results and discussion}

We developed a fluorophore-spacer-receptor system (I) ${ }^{15}$ comprising 4-amino-1,8naphthalimide (ANP) moiety as the fluorophore, a dimethylamino moiety as the receptor and a $-\mathrm{CH}_{2}-\mathrm{CH}_{2}-$ moiety as the spacer. However, the off-on fluorescence signalling efficiency of $\mathbf{I}$ was found to be rather poor and we could observe only $1 \cdot 1-1 \cdot 3$-fold enhancement of the fluorescence of the system in the presence of the first row of the transition metal ions. The oxidation potential of the fluorophore component, which is a measure of the electron-richness of the fluorophore is estimated to be 1.27 V. Since according to our design logic an electronically deficient fluorophore is a better suited fluorophore component for the required fluorophore-spacer-receptor system, we developed II, ${ }^{15}$ a system structurally very similar to I but contains 4 -aminophthalimide (AP) moiety as the fluorophore instead of ANP used in the previous case.

The oxidation potential of AP $(1.50 \mathrm{~V})$ is measured to be higher than that of ANP confirming the electron deficient character of the former. We also noted that in a given solvent, AP emits at a higher energy compared to ANP. The free energy change associated with the photoinduced intramolecular electron transfer (PIET) process is given by, $\Delta G=E_{\mathrm{ox}}-E_{\mathrm{red}}-E_{\mathrm{s}},{ }^{20}$ where $E_{\mathrm{ox}}$ and $E_{\mathrm{red}}$ are the oxidation potential of the donor (receptor) and the reduction potential of the acceptor (fluorophore) moiety respectively and $E_{\mathrm{s}}$ is the energy of the fluorescent state of the molecule. Since the $E_{\text {red }}$ and $E_{\mathrm{s}}$ values (vide table 1) of AP are more favorable for an efficient PIET in II, one expects II to be a 
<smiles>CNCCN1C(=O)c2cccc3c(N)ccc(c23)C1=O</smiles>

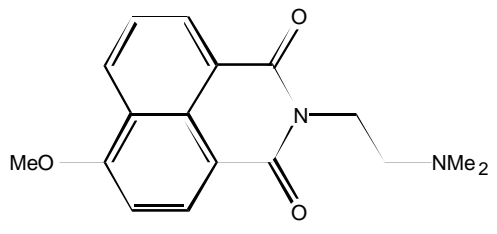

III<smiles>CNCCN1C(=O)c2ccc(N)cc2C1=O</smiles>

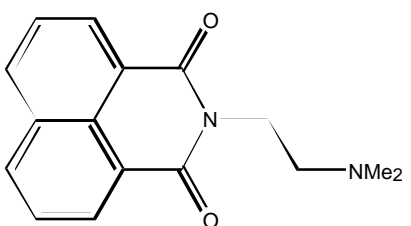

IV<smiles>CNCCN1C(=O)c2cccc3c(Cl)ccc(c23)C1=O</smiles>

Chart 1.

better fluorosensor than I. The $\Delta G$ values associated with PIET in I and II are calculated to be -6.6 and $-12.7 \mathrm{kcal} / \mathrm{mol}$ in acetonitrile. The off-on signalling efficiency of II, as measured by the FE of the system in the presence of various metal ions, is found much higher than that exhibited by I. The FE values observed for II in the presence of the various transition metal ions lie between 34 and 55 (vide table 2).

A typical variation of the fluorescence output of II in the presence of the metal ion is illustrated in figure 1. While the fluorescence enhancement is due to suppression of PIET in the presence of metal ions, the red shift of the fluorescence maximum is due to metal ion induced enhanced charge separation in the fluorophore moiety.

Since a methoxy group is a poorer electron donor compared to an amino group, 4methoxy-1,8-naphthalimide (MNP) is expected to be electronically deficient compared to ANP and hence, according our design logic, III that comprises MNP as the fluorophore should be a better fluorosensor for the transition metal ions compared to I. A study of the fluorescence behaviour of III in the presence of the various d-block elements does indicate that this system shows much better fluorescence signalling properties ${ }^{16}$ compared to $\mathbf{I}$. The FE values observed with this system in the presence of various transition metal ions in acetonitrile vary between 15 and 28 (vide table 2).

As the next step, we thought about an unsubstituted 1,8-naphthalimide (NP) moiety as the fluorophore component. The absence of the amino or the methoxy group at the 4position is expected to make this fluorophore component electronically deficient and suitable for sensing purpose. A study of the redox behaviour shows that NP does not 
Table 1. Redox potential ${ }^{\mathrm{a}}$ and energy of the emitting state ${ }^{\mathrm{b}}$ of the various fluorophores used in the sensor systems. Calculated free energy changes ${ }^{c}$ associated with PIET in the multi-component systems are also shown.

\begin{tabular}{lccccc}
\hline Fluorophore & $E_{\text {ox }}(\mathrm{V})$ & $E_{\text {red }}(\mathrm{V})$ & $E_{\mathrm{s}}(\mathrm{kcal} / \mathrm{mol})$ & Sensor & $\Delta G(\mathrm{kcal} / \mathrm{mol})$ \\
\hline ANP & 1.27 & -1.61 & 55.04 & $\mathrm{I}^{\mathrm{e}}$ & -6.6 \\
AP & 1.50 & -1.66 & 62.2 & II $^{\mathrm{e}}$ & -12.7 \\
MNP & $-{ }^{\mathrm{d}}$ & -1.11 & 65.3 & III $^{\mathrm{f}}$ & -28.4 \\
NP & $-{ }^{\mathrm{d}}$ & -1.00 & 79.0 & IV $^{\mathrm{f}}$ & -44.6 \\
\hline
\end{tabular}

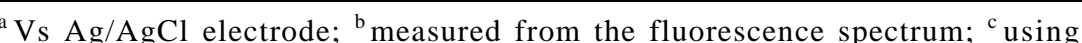
$\Delta G=E_{\mathrm{ox}}-E_{\mathrm{red}}-E_{\mathrm{s}} ;{ }^{\mathrm{d}}$ no oxidation of the fluorophore could be observed between 0 and $2 \mathrm{~V} ;{ }^{\mathrm{e}}$ ref $15 ;{ }^{\mathrm{f}}$ ref 16

Table 2. Off-on signalling efficiency, as measured by the fluorescence enhancement ${ }^{\mathrm{a}}$ of the various sensor systems in the presence of $\mathrm{Zn}^{2+}$ and $\mathrm{Cr}^{3+}$ in tetrahydrofuran.

\begin{tabular}{lcc}
\hline System & Maximum FE with $\mathrm{Zn}^{2+}$ & Maximum $\mathrm{FE}$ with $\mathrm{Cr}^{3+}$ \\
\hline $\mathrm{I}^{\mathrm{b}}$ & $1 \cdot 3$ & $1 \cdot 1$ \\
$\mathrm{II}^{\mathrm{b}}$ & 55 & 39 \\
$\mathrm{III}^{\mathrm{c}}$ & 13 & 8 \\
$\mathrm{IV}^{\mathrm{c}}$ & 2150 & 350 \\
$\mathrm{~V}^{\mathrm{d}}$ & 735 & 185 \\
\hline
\end{tabular}

${ }^{a}$ Fluorescence intensity of the system in the presence of the metal ion relative to that of the same system in the absence of the metal ion; ${ }^{b}$ ref $15 ;{ }^{c}$ ref $16 ;{ }^{d}$ ref 17

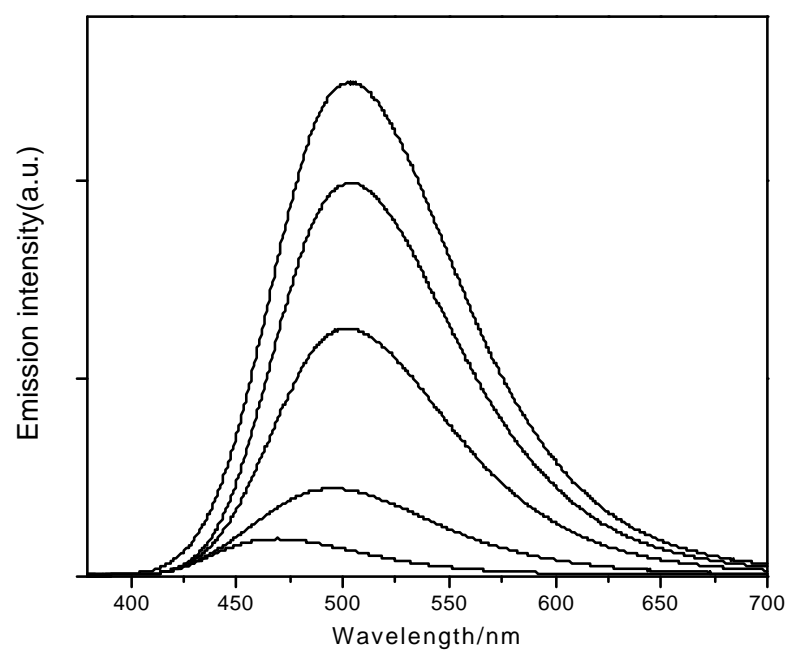

Figure 1. Fluorescence spectra of II in acetonitrile in the presence of $\mathrm{Zn}^{2+}$. The concentrations of the metal ion in increasing order of the fluorescence intensity are 0 , $5.2 \times 10^{-6}, 2 \cdot 1 \times 10^{-5}, 4.2 \times 10^{-4}$ and $1.4 \times 10^{-3} \mathrm{M}$ respectively. 
exhibit any oxidation between $0-2 \mathrm{~V}$ thereby supporting our assumption that NP is expected to be superior fluorophore component for the design of the fluorophore-spacerreceptor system. The energy corresponding to the $0-0$ transition from the first excited state of NP, as estimated from its emission spectrum is $79 \mathrm{kcal} / \mathrm{mol}$. A higher singlet state energy of NP is largely responsible for a highly negative $\Delta G$ value $(-44.6 \mathrm{kcal} / \mathrm{mol})$ for PIET in the multi-component system comprising NP as the fluorophore, IV. ${ }^{16}$ Interestingly, IV, though exhibits high fluorescence enhancement, the FE values observed for this system in the presence of quenching metal ions are found to be much higher than the expected values. The maximum value of FE expected for a given system is essentially the ratio of the fluorescence quantum yield $\left(\phi_{f}\right)$ of a fluorophore to that of the corresponding fluorophore-spacer-receptor system. Since this ratio is $\sim 40$, one expects, assuming complete recovery of fluorescence in the presence of metal ions, only 40-fold enhancement of the fluorescence intensity of IV. Interestingly, the FE value observed for this system is 50 -fold higher than the expected value. This unusual behaviour of the system was traced largely due to the solvent sensitivity of the fluorescence quantum yield of the fluorophore, NP. ${ }^{16}$ The measured $\phi_{f}$ of NP is 0.24 in water and $1.1 \times 10^{-2}$ in nonpolar tetrahydrofuran. Since the transition metal ions were added in the form of the hydrated salts of the metals, we realized that it is possible to observe FE of the system by an additional factor of 22 (which is the ratio of the fluorescence quantum yield of the fluorophore in the two solvents stated above) just due to a change of the microenvironment around the fluorophore. That this is one of the most likely reasons why IV exhibits a much higher FE value than that expected on consideration of PIET in the system is evident from the fact that III, which is a structurally similar system but does not contain a fluorophore with solvent-sensitive fluorescence properties, exhibits a normal behaviour. On the other hand, V, which contains fluorophore (4-chloro-1,8naphthalimide) with solvent-sensitive properties, exhibits unusually high fluorescence enhancement similar to that shown by $\mathbf{I V} .^{17}$

\section{Conclusion}

A design strategy for the fluorosensors for the transition metal ions based on an electronically deficient fluorophore component is suggested. It is shown that a simple fluorophore-spacer-receptor system can be used for off-on signalling of the transition metal ions well-known for their fluorescence quenching abilities when the right fluorophore component is chosen for the construction of the multi-component system.

\section{Acknowledgements}

We thank the Department of Science and Technology, Government of India and the Council of Scientific and Industrial Research (CSIR), New Delhi for financial support. Thanks are also due to Drs B Ramachandram and G Saroja for contributing to the development of some of the sensor molecules. NBS and SB thank the CSIR for fellowships.

\section{References}

1. Carter F L, Siatkowski R E and Wohltjen H 1988 Molecular electronic devices (Amsterdam: Elsevier)

2. Lehn J M 1995 Supramolecular chemistry (Weinheim: VCH) 
3. Lehn J M 1988 Angew. Chem., Int. Ed. Eng. 2789

4. de Silva A P, Gunaratne H Q N, Gunnlaugsson T, Huxley A J M, McCoy C P, Rademacher J T and Rice T E 1997 Chem. Rev. 971515

5. de Silva A P, Gunaratne H Q N and McCoy C P 1993 Nature (London) 36442

6. Bissel R A, de Silva A P, Gunaratne H Q N, Lynch P L M, Maguire GEM and Sandanayake K R A S 1992 Chem. Soc. Rev. 21187

7. de Silva A P, Gunaratne H Q N and McCoy C P 1997 J. Am. Chem. Soc. 1197891

8. Czarnik A W 1993 Fluorescent chemosensors for ion and molecule recognition (Washington, DC: Am. Chem. Soc.)

9. Balzani V 1987 Supramolecular photochemistry (Dordrecht: Reidel)

10. Bissel R A, de Silva A P, Gunaratne H Q N, Lynch P L M, Maguire G EM, McCoy CP and Sandanayake K R A S 1993 Top. Curr. Chem. 168223

11. Fabbrizzi L and Poggi A 1995 Chem. Soc. Rev. 24197

12. Prodi L, Bolletta F, Montalti M and Zaccheroni N 2000 Coord. Chem. Rev. 20559

13. Montalti M, Prodi L, Zaccheroni N, Charbonnière L, Douce L and Ziessel R $2001 \mathrm{~J}$. Am. Chem. Soc. 12312694

14. Ghosh P, Bharadwaj P K, Mandal S and Ghosh S 1996 J. Am. Chem. Soc. 1181553

15. Ramachandram B and Samanta A 1997 J. Chem. Soc., Chem. Commun. 1037

16. Ramachandram B, Saroja G, Sankaran N B and Samanta A 2000 J. Phys. Chem. B104 11824

17. Ramachandram B, Sankaran N B, Karmakar R, Saha S and Samanta A 2000 Tetrahedron 7041

18. Kemlo J A and Shepherd T M 1977 Chem. Phys. Lett. 47158

19. Varnes A W, Dodson R B and Wehry E L 1972 J. Am. Chem. Soc. 94946

20. Weller A 1968 Pure Appl. Chem. 16115 